\title{
Displays and Photonics Application Group Activities
}

\author{
C. VÁZQUEZ \\ J. M. S. PENA \\ S. VARGAS \\ I. PÉREZ
}
A. B. GONZALO
V. URRUCHI
R. VEGAZ
J. MONTALVO
R. MANZANARES
J. I. SANTOS

A. L. ARANDA

Departamento Tecnología Electrónica

Universidad Carlos III

Madrid, Spain

\begin{abstract}
Displays and Photonics Application Group (GDAF) is devoted to design and development of passive and active devices and sensors for their applications in WDM networks, CWDM, POF, and RF over fiber networks using different technologies such as optical fibers, liquid crystals, and integrated optics. The group is also very active in modeling, electrooptical characterization, and addressing electronics of LCDs. In this article we are going to describe the main activities carried out in the group.
\end{abstract}

Keywords optical fibers, integrated optics, liquid crystals, sensors, filters, displays

\section{Research Group Background}

Displays and Photonics Application Group (Grupo de Displays y Aplicaciones Fotónicas, GDAF) is made of people coming from different photonics and electronics areas. They have been working with optical fibers, integrated optics, liquid crystals, and related topics, from integrated optics devices design and characterization in III-V compounds such as InP and InGaAsP for application in optical transparent networks in the frame of European projects [1,2] to design and characterization of novel tunable filters [3] and recirculating 
delay lines based on erbium-doped fibers [4]. Concerning liquid crystals, modeling, electrooptical characterization, addressing electronics, and different photonics applications have been developed in the frame of European projects [5-7] and thematic networks [8, 9].

In the last years, we have also developed low-cost sensors and components based on optoelectronic devices and plastic optical fibers as part of sensor and local area networks for industrial and disabled people applications. Some networks to be funded by the European Commission are now under evaluation [10].

\section{Research Fields}

GDAF members have been and are currently involved in research projects encompassing different areas within the field of optical communications and photonics. The Group is mainly public funded (European Union, Spanish, and Regional Governments) although it has some private incomes from different Spanish companies. The current research interests include the following areas:

- Passive and active devices and their applications in WDM networks, CWDM and RF over fiber. Design and development of filters and demultiplexers using innovative resonators structures in combination with cheaper integrated amplifiers. Optical filter synthesis based on digital filter techniques is also carried out. Filter specifications are optimized depending on the network. Experience in designing photonic integrated circuits (PIC) and passive devices based on multimode interference principles (MMI) is also used.

- Passive and active devices and their applications in plastic optical fiber (POF) networks. We develop passive components such as couplers, power dividers, and shutters, and we combine liquid crystals (LC) with POF to design active optical couplers, modulators, and switches. Operating not only in the visible region but also in $850 \mathrm{~nm}$ and $1300 \mathrm{~nm}$ with graded-index POF for broadband local area networks with distances up to hundreds of meters in sectors such as automation, automotive, and home networks. Switches experiments on the $1550 \mathrm{~nm}$ window with other fibers are under development. Other photonics applications of LC are also developed (routing devices, only phase modulators, panelled windows, etc.) in visible and near IR ranges.

- Optical sensors and POF sensors. Optical technologies with optical fibers are intrinsically safe in nature, with no risk of explosion even under malfunction operation, because optical fibers are inert materials. We develop full prototypes of different low-cost POF sensors (e.g., level sensors, gas sensors) and their integration in sensor and local area networks to be used in industrial environments with inflammable atmospheres (e.g., petrol stations) and in vehicles (e.g., cars, planes). Concerning other optical sensors, sensors for application in rehabilitation technology have been developed.

- Modeling, electrooptical characterization and addressing electronics of LCDs. Different LCD technologies have been studied in the last years. From polymer/gelglass-dispersed liquid crystals (PDLCs/GDLCs), twisted nematic (TN), ferroelectric, and antiferroelectric passive matrix displays to TN active matrix displays (AMLCDs), a set of theoretical models, electro-optical characterization protocols, and electronic drivers have been developed by the Group. Currently, new chiral smectic liquid crystals are being explored in order to predict potential applications for high-end displays (full color, grey scale, video rate). 


\section{Relevant Results and Prototypes}

In this section we are going to describe some of the results and research activities developed or under development in GDAF.

\section{Filters and Demultiplexers}

We have designed and developed different filters and demultiplexers for DWDM networks having high crosstalk and flexibility by using active configurations based on ring resonators (RR) [11]. We have tried to overcome constraints related to minimum bend losses such as the limit in the maximum free spectral range of these devices. Some of our novel devices break the periodicity of the transfer function using Bragg gratings [3], while others mix Mach-Zehnder interfometer (MZI) stages with RR [12]. Those filters can also be used for extracting subcarrier multiplexed (SCM) data channels in DWDM. More recently, devices based on a loop mirror in a ring resonator, which can be tuned by changing the coupling coefficient of a directional coupler, have been developed. The loop mirror is implemented using Bragg gratings in a transmission mode $[13,14]$ or a Sagnac configuration [15] to have a perfect symmetry between the signals to be interfered (copropagating and counter-propagating ones). The filter structure allows optical integration for having higher free spectral ranges. Measurements on a passive, optical fiber tunable filter are developed to validate the models. On the other hand, we use digital filter processing techniques [16-18] for developing filters with controlled characteristics in terms of pass bandwidth, stop band rejection, and the transition width between the pass band and the stop band. In these designs we used second-order systems implemented with different configurations that only used a single RR. In Figure 1a the general structure can be seen (Figure 1a) and two specific designs using Bragg gratings in a transmission mode (see Figure 1b) and a Sagnac or loop mirror (see Figure 1c). The second configuration has better properties to be used in the synthesis process since it has a wider angular pole location in the $z$ plane. The experimental setup shown in Figure 2 has been used to characterize the filter's responses [15], in collaboration with the Fiber Optics Metrology Group of the Consejo Superior de Investigaciones Científicas; another version of this setup is now operative in our laboratories and is described later under Installations.

We have also designed modal filters to be used in integrated passive and active devices based on MMI principles. This is a useful device when for different reasons (such as monolithic integration restrictions, photolithography, and/or coupling alignments tolerances) the first-order mode is excited in the access waveguides of the PIC. The impact of first-order mode excitation on $3 \mathrm{~dB}$ restricted interference MMI couplers [19, 20] depending on alignment conditions has been quantified and measured in our group in collaboration with the Public University of Navarra [21, 22]. A photograph of the $3 \mathrm{~dB}$ MMI couplers, based on buried InP/InGaAsP waveguides, which have been used in the measurements, is shown in Figure 3 [1, 2].

In all the design work developed in relation to the previous topics, we use home-made software tools and models, along with other commercial software tools (e.g., MATLAB, VPI Transmission \& Component Maker, BPM).

\section{Optical Sensors}

Different prototypes of optical sensors have been developed for applications in industry and the disabled people community. 
(a)

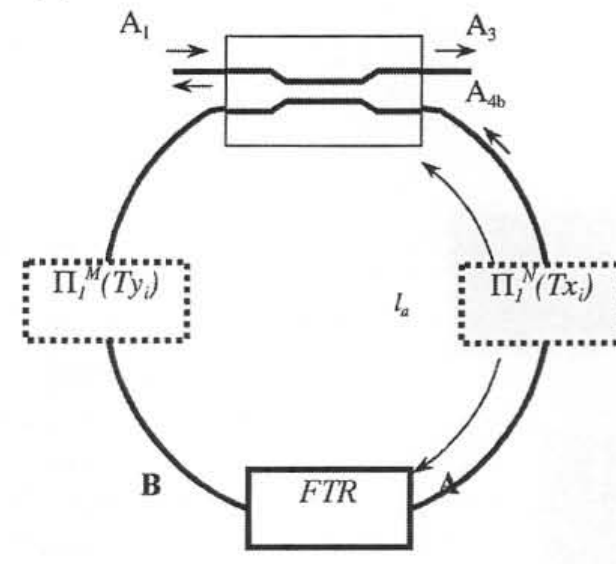

(b) Bragg

A

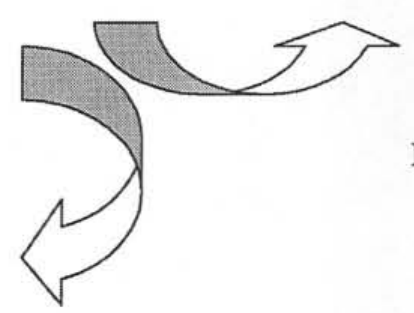

A

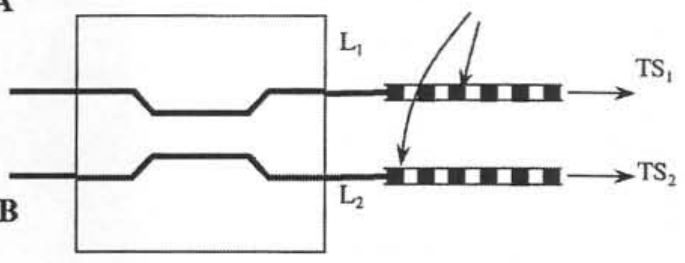

(c)

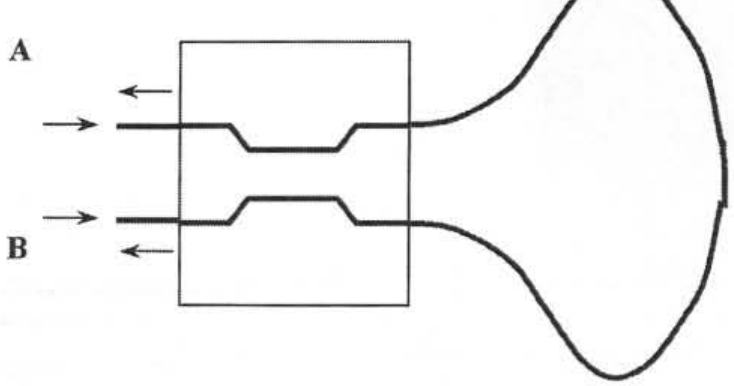

Figure 1. (a) A schematic of the general filter based on multireflection functions in the RR, two specific designs: (b) with Bragg gratings in a transmission mode, (c) with a Sagnac or loop mirror.

Special mention is devoted to a level sensor based on plastic optical fibers to be used in critical environments such as inflammable atmospheres [23, 24]. A schematic of the multi-sensor, scalable system can be seen in Figure 4a. Optical fibers are used in the sensor heads and for optical multiplexing to address different tanks without using multiple cables. This system allows an intrinsically safe, level measurement with a simple, modular, and cost-effective solution where the multiplexed signals are transmitted to the PC located in the central office (server). A fiber-optic local area network to connect the server and different clients (other PCs) using an optical fiber active star configuration is also developed. The same user-friendly interface is installed in the different PCs of the network for monitoring in real time the level and the alarms status in the different tanks. A full calibration is carried out and a good linearity better than $1.5 \%$ FS (full scale) is obtained in a $2 \mathrm{~m}$ range. Our test bench, which can be seen in Figure $4 \mathrm{~b}$ along with 


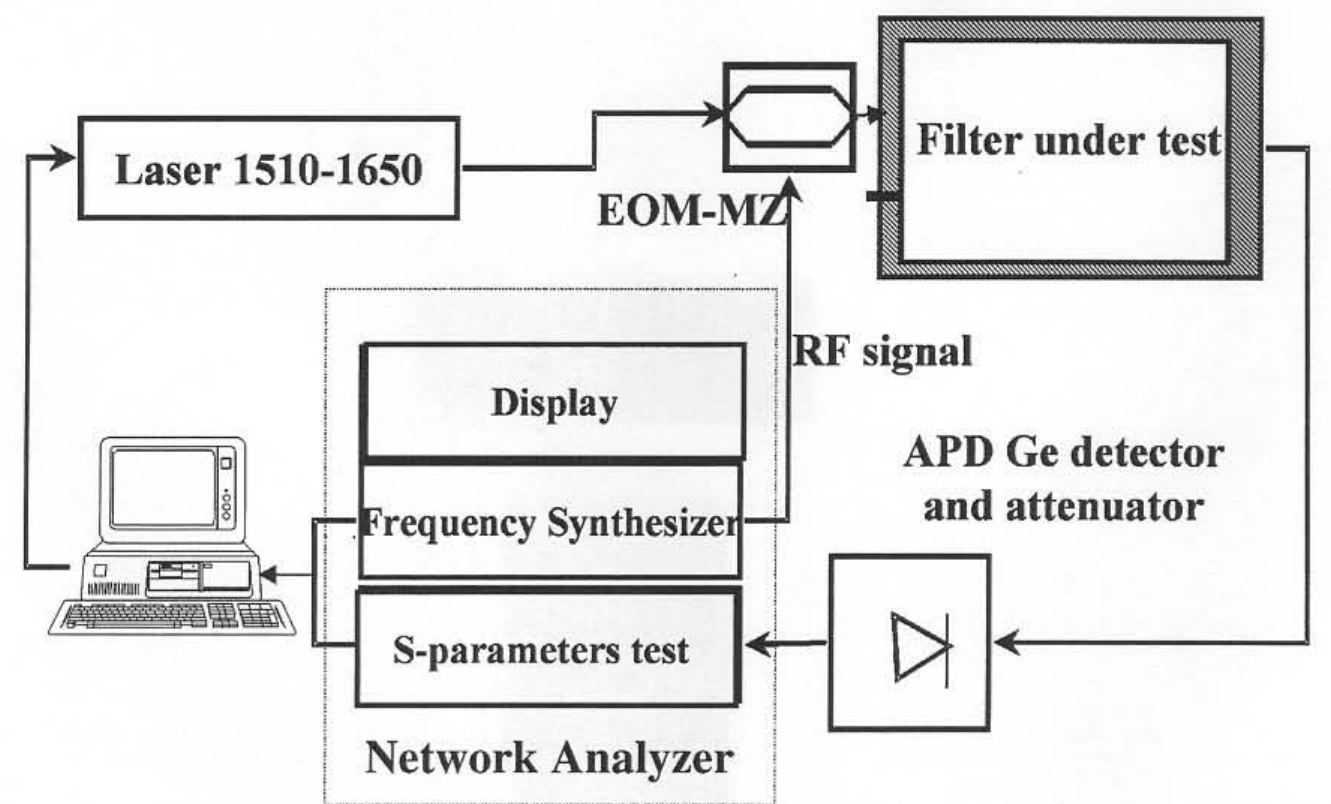

Figure 2. Diagram of the setup used for measuring the filter response under coherent regime.

two different sensor heads, has limited the measured sensor range. Resolution better than $0.5 \%$ FS is obtained and can be improved employing a better conditioning circuit. All the electronics and optoelectronics have been developed in the group (see Figure 5). A theoretical model is also under development and has already been used for optimizing the sensor head parameters in future prototypes.

Other level sensors with shorter ranges, tenths of centimeters, have been developed using POFs, along with control level sensors based on light detector resistances [25].

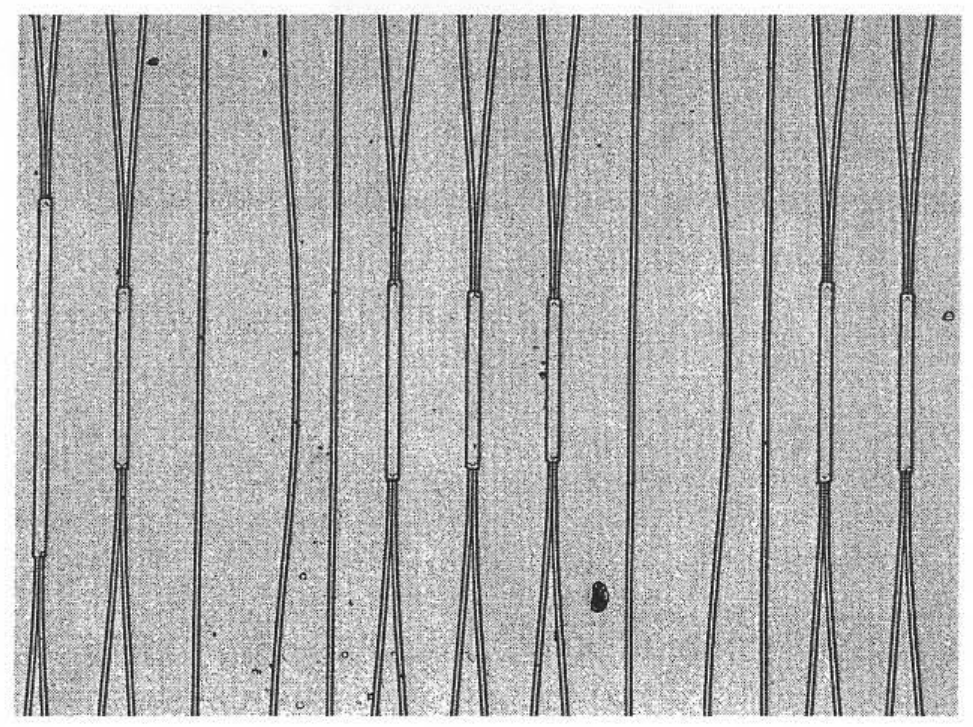

Figure 3. A photograph of the fabricated MMI $3 \mathrm{~dB}$ restricted interference couplers measured to determine the impact of excitation on first-order mode in accessing waveguides. 


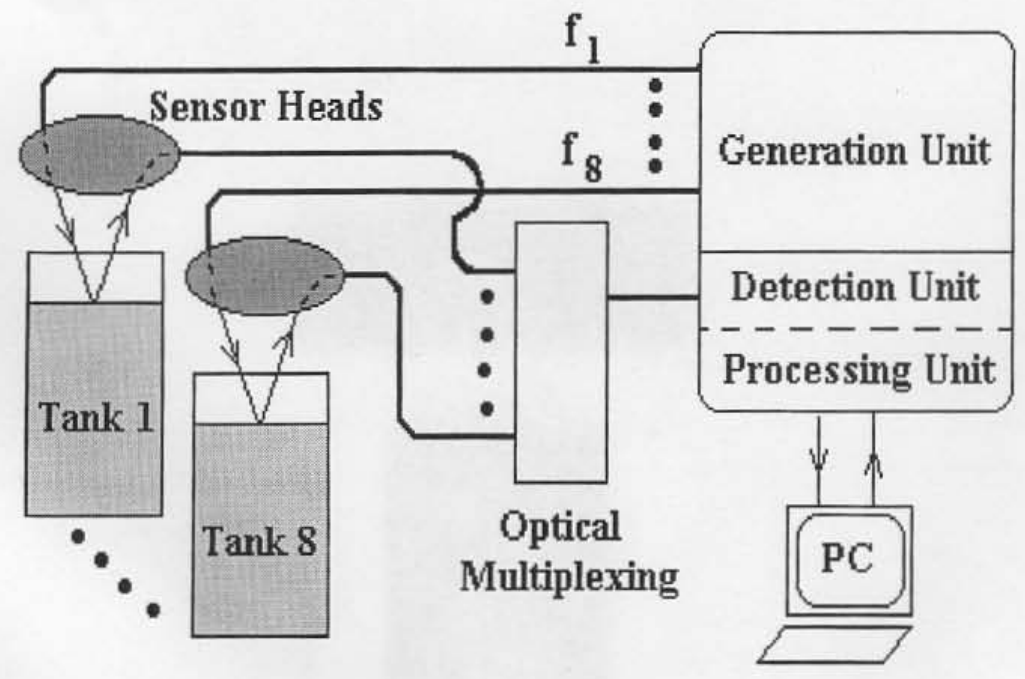

(a)

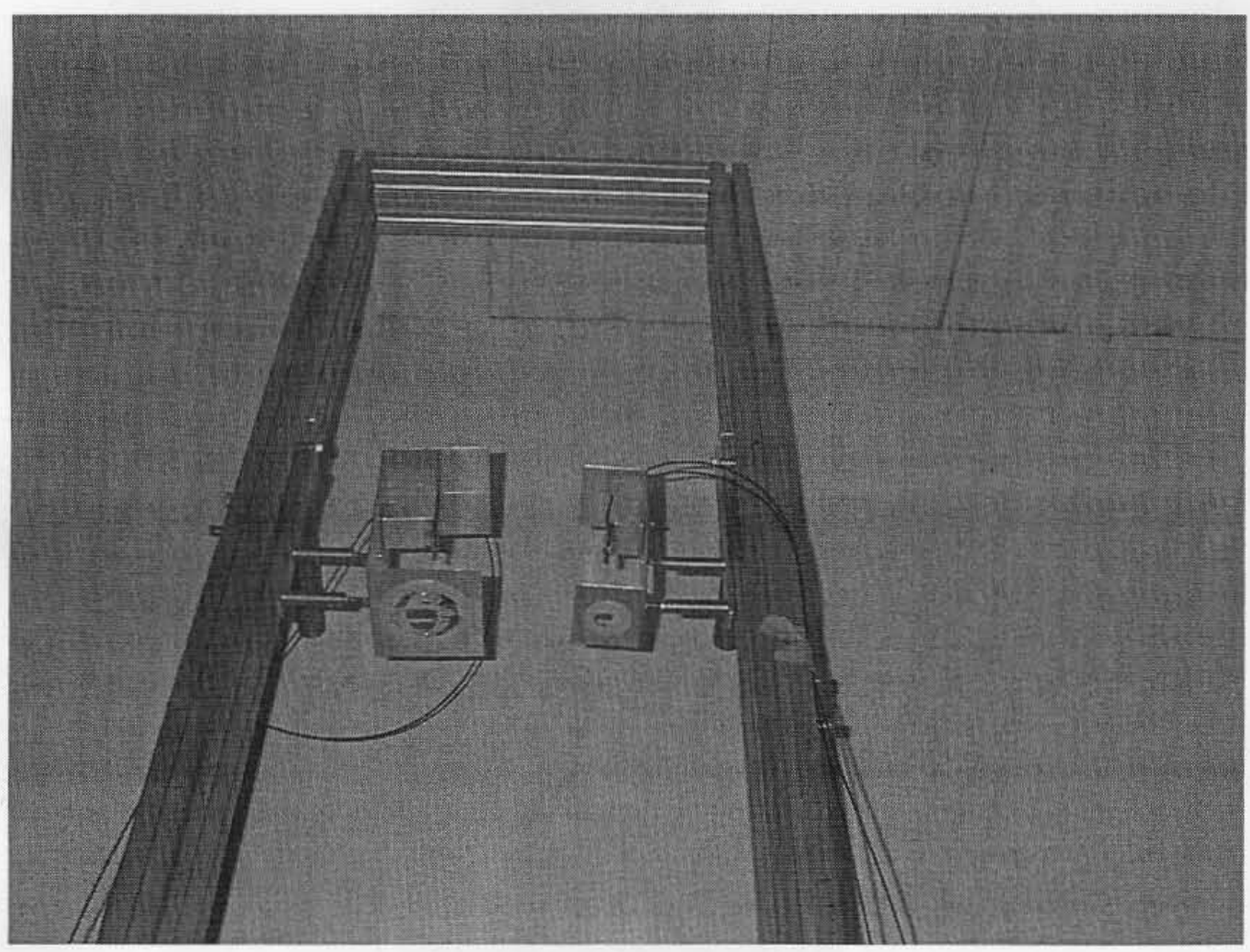

(b)

Figure 4. (a) A schematic of the multi-sensor system, (b) a photograph of the test bench, including POF sensor heads, used in the calibration of the level multi-sensor system. 


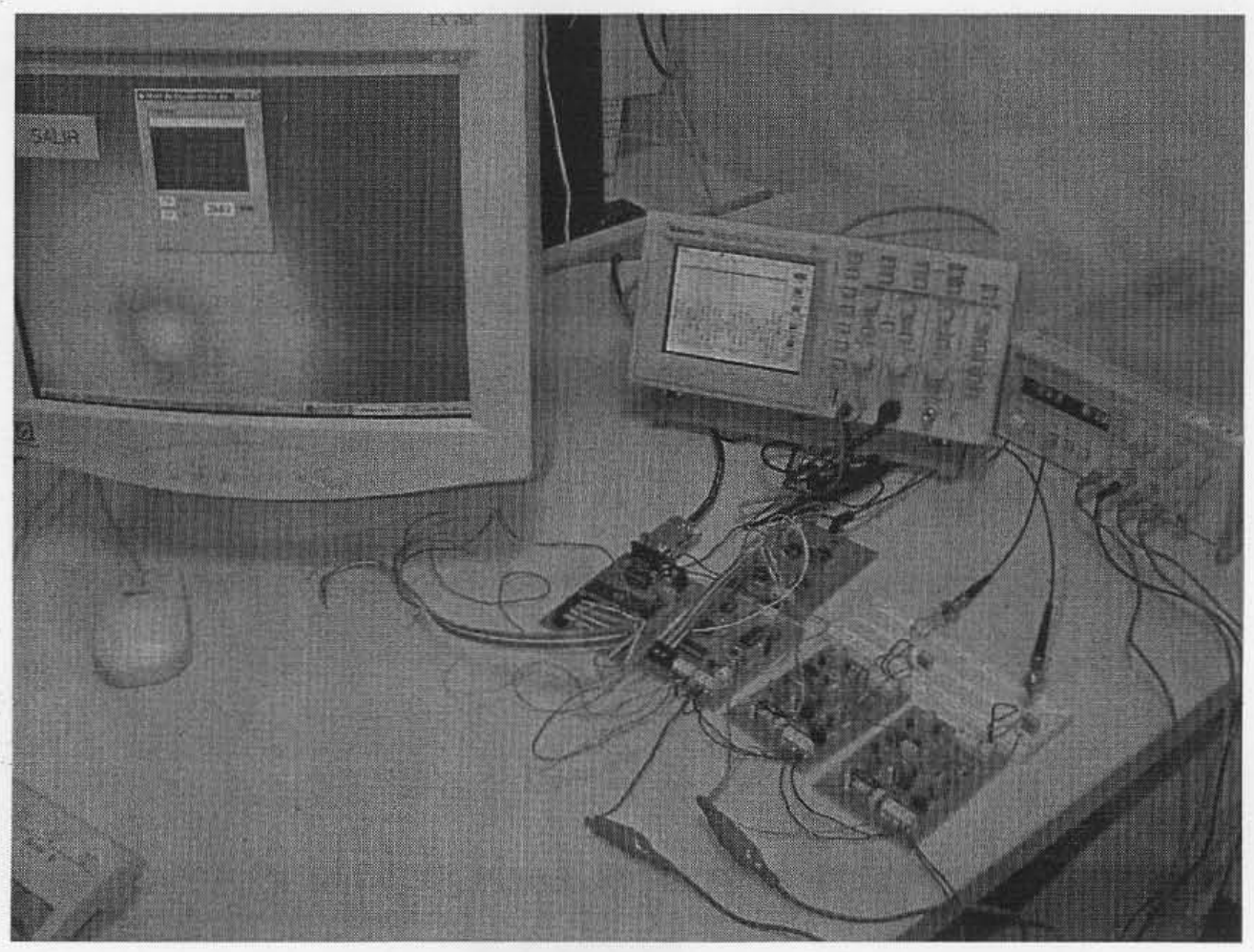

Figure 5. A photograph of the different PCBs developed for controlling the level multi-sensor system: a unique PCB for the reception circuit, and 1 PCB of each sensor head driver.

On the rehabilitation technology, a microcontrolled, optoelectronic, portable, color identifier for visually impaired people has been implemented [26]. It is based on the detection of the reflected optical light coming from the target whose color it is intended to find out. Three primary LEDS (R, G, B) sequentially emit the optical sensing signal. A photograph of the developed prototype can be seen in Figure 6.

\section{LC Switches and Other LC Photonics Applications}

For the different devices mainly oriented to POF networks that we have developed [27] we area going to describe the LC switches that combine fiber-optic and liquid crystal technologies. Perfluorinated (PF) polymer-based GI-POF (graded index polymer optical fibers) has a low loss wavelength region from 500 to $1300 \mathrm{~nm}$, allowing implementation of coarse WDM in high-speed, reconfigurable POF networks and in fiber-optic sensor networks [28]. We have developed compact, scalable, broadband, high interchannel crosstalk fiber-optic switch configurations, using nematic LC (NLC) cells for those networks. These NLC require low power levels for working and use a display technology well established and with a large-scale capability. The high numerical aperture and high diameter of POFs reduces cost connections and losses. The simplest proposed configuration is reported in Figure 7a, and a photograph of the developed prototype can be seen in Figure $7 \mathrm{~b}$. This prototype includes all the electronics for a specific application as a switch connecting two redundant paths in a sensor network [29]. Measurements of the 


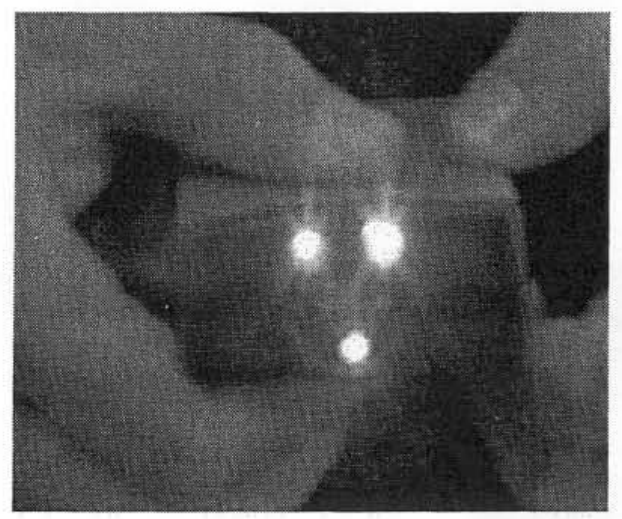

(a)

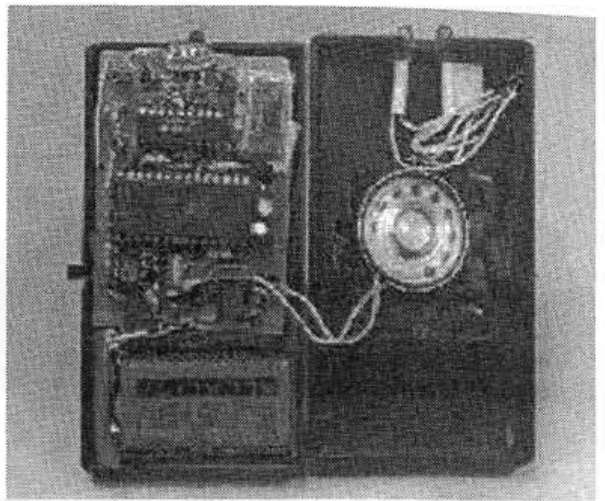

(b)

Figure 6. Photographs of the device: (a) R-G-B LEDs emitting the optical signals and (b) inner view showing the electronic components used.

simultaneous performance of the NLC at $650 \mathrm{~nm}$ and $850 \mathrm{~nm}$, showing its capability for switching at both wavelengths, have already been performed.

We are also working in implementing $2 \times 2$, polarization-insensitive broadband switches. In these $2 \times 2$ switches, both polarizations of the input light are separated with a PBS and are processed independently.

Another prototype that has been developed based on another LC technology is an electro-optic system based on a micro-controlled driver for on-line control of PDLC windows [30]. A photograph of the device under operation can be seen in Figure 8. Only the visible light is regulated. The system includes two working modes. In the "feedback mode," a lighting level selected by the user is maintained constant modifying the window transparency, according to variations of external illumination upon time. In

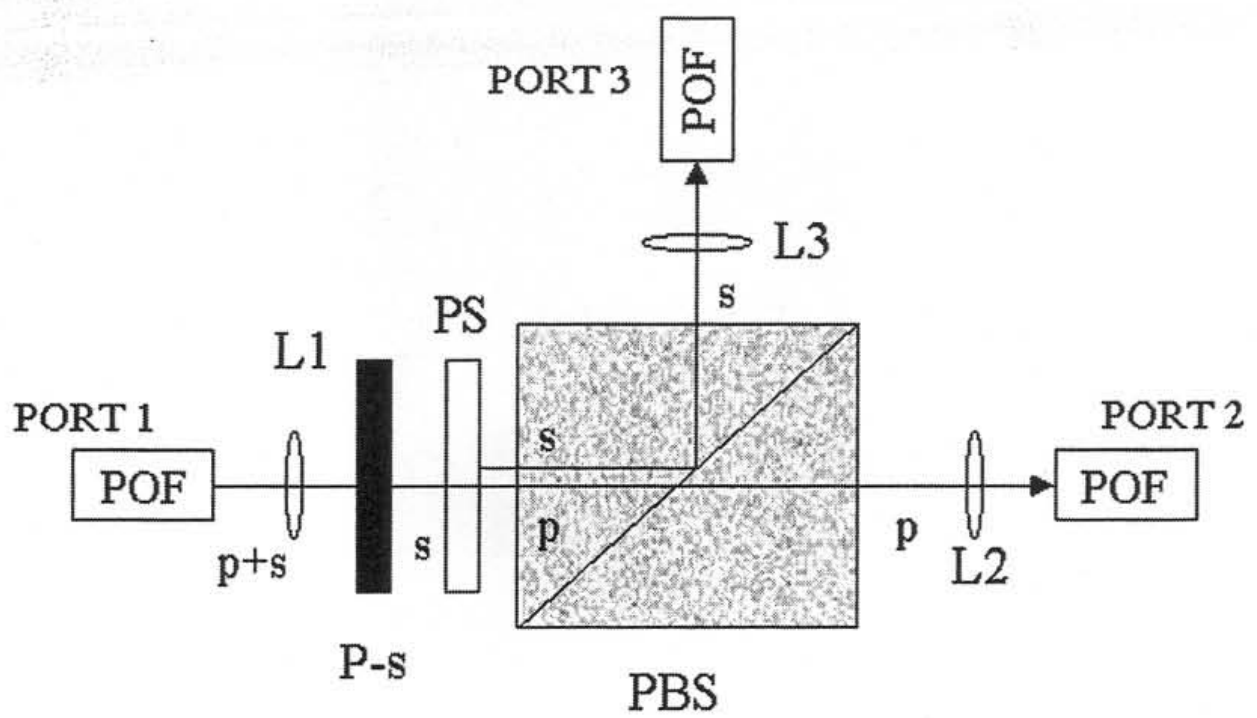

(a)

Figure 7. (a) A schematic of the simplest $1 \times 2$ NLC switch. 


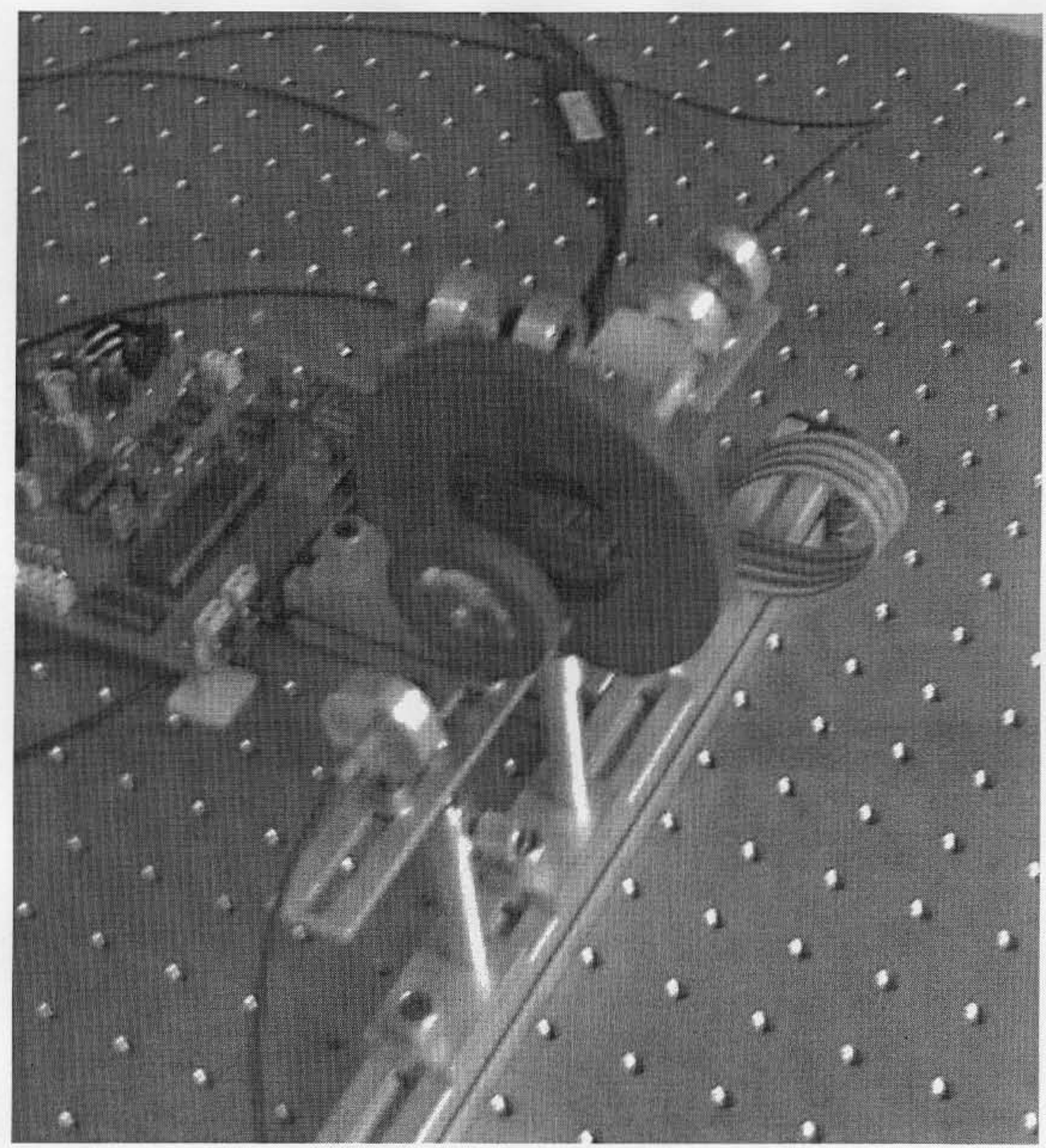

(b)

Figure 7. (b) A photograph of the implemented prototype with the control electronics.

the "fixed mode" the user selects a fixed transparency of the window, regardless the illumination. This mode is intended for indoor panels, e.g., using the PDLC panel as a projection screen or to isolate a panelled room for temporary privacy.

\section{Electro-Optical Characterization and Electronic Drivers for LCDs}

Some characterization protocols based on a set of experimental measurements are developed by GDAF in order to evaluate the electro-optical performance as well as the dynamic behavior of the different technologies of LCDs. Characteristics such as electrical and optical hysteresis cycles (see Figure 9), switching currents, and ionic effects, among others, are usually checked in order to evaluate the electro-optical performance 